PEDRO SOZA R.

Profesor Asistente

Departamento de Arquitectura

Facultad de Arquitectura y Urbanismo

Universidad de Chile

Desde Atlanta, USA

\section{Creatividad sistémica}

Tal como es sugerido por el título de esta publicación, desde las perspectivas del diseño computacional y de la cognición del diseño, el presente número de revista de arquitectura ofrece una serie de artículos que en su conjunto pretenden aproximarnos al estado del arte en investigación científica y sistemática, de carácter tanto internacional como local, que persigue incrementar nuestro entendimiento y conocimiento en distintas áreas del saber, que forman parte del fenómeno creativo en la práctica del diseño arquitectónico.

Los tres primeros artículos, de Kasaly, Gómez y Abdelmohsen ofrecen, desde la perspectiva de la investigación científica, tres aproximaciones al uso de ciencia y tecnología para informar los procesos de diseño arquitectónico. Kasaly, constatando que los arquitectos no solemos movernos fuera de nuestras propias convicciones para tomar decisiones de diseño, presenta un estudio observacional «in-situ» de equipos interdisciplinarios, desarrollando un proyecto arquitectónico para un hospital rural y discute cómo los miembros del equipo de diseño no solo toman decisiones de diseño sino que además construyen evidencia que sustente dichas decisiones. Gómez por su parte da cuenta del rol que desempeñan los análisis espaciales computacionales actuales para soportar la toma de decisiones proyectual. Sobre esa base plantea su propio trabajo de investigación centrado en la ocupación espacial que realizan las personas del espacio como variable proyectual. El artículo propone un método para implementar dicho tipo de análisis y expone los resultados de un estudio exploratorio hecho por los autores al respecto. Abdelmoshem cierra este primer grupo exponiendo un marco de trabajo para implementar un sistema computacional de chequeo automático de reglas y patrones de circulación en exteriores de edificios, concretamente un método de revisión automático de circulaciones de acuerdo a reglas propuestas por el arquitecto, como podría ser por ejemplo la normativa legal vigente, en ambientes BIM.

A continuación, los trabajos de Valdez y Celani ofrecen al lector el estado actual de la Fabricación Digital y el relato de la experiencia de un laboratorio de investigación en fabricación digital con foco en la docencia en Brasil. En particular, Valdez aborda el estado actual de la fabricación Digital, su relación con la industria del diseño y arquitectura, y propone una proyección al año 2030 sobre el impacto que esta tecnología puede tener y las barreras que aún quedan por trabajar para que dicho impacto sea positivo en la industria AEC (Arquitectura, Ingeniería, Construcción). Celani, en el relato sobre el laboratorio LAPAC, ofrece una mirada retrospectiva después de siete años de trabajo en investigación y docencia con alumnos de las escuelas de Arquitectura, Ingeniería Civil y Diseño Urbano y ofrece conclusiones de dicha experiencia tanto en docencia como en investigación.

De manera similar, los trabajos de Cox y Gurovich, Fernández, y de Rossel y Loyola exponen parte del panorama actual en la FAU de la Universidad de Chile. En particular, el artículo de Cox y Gurovich expone los resultados del curso de simulaciones urbanas que han realizado en la FAU con especial enfoque en métodos de modelamiento urbano mientras que discuten criterios de evaluación y perspectivas futuras. Fernández a su vez nos presenta un proyecto de investigación arquitectónico centrado en el uso de espacios urbanos de pequeña escala como dispositivos de memoria e información que podrían contribuir a la toma de decisiones proyectuales. Rossel y Loyola cierran la tercera parte del presente número ofreciendo una sólida crítica a la contradicción entre el uso de tecnologías digitales en el ámbito del diseño arquitectónico y el uso de argumentos racionalista para justificar arbitrariedades formales, sin reconocer los aspectos subjetivos y emocionales involucrados en la toma de decisiones proyectual.

Finalmente, y en un intento por entender precisamente la operatoria en la selección y uso de algunos argumentos por sobre otros en la toma de decisiones proyectuales, mi propio trabajo ofrece una mirada al fenómeno creativo desde la interacción de tres de los componentes cognitivos que operan a nivel mental cuando los arquitectos tomamos decisiones de diseño: la imaginería visual, la percepción visual y el conocimiento semántico que los arquitectos desarrollamos y usamos para llevar adelante los procesos de diseño. 